\title{
WASP-80b: a gas giant transiting a cool dwarf ${ }^{\star \star \star}$
}

\author{
A. H. M. J. Triaud ${ }^{1}$, D. R. Anderson ${ }^{2}$, A. Collier Cameron ${ }^{3}$, A. P. Doyle ${ }^{2}$, A. Fumel ${ }^{4}$, M. Gillon ${ }^{4}$, C. Hellier ${ }^{2}$, \\ E. Jehin ${ }^{4}$, M. Lendl ${ }^{1}$, C. Lovis ${ }^{1}$, P. F. L. Maxted ${ }^{2}$, F. Pepe ${ }^{1}$, D. Pollacco ${ }^{5}$, D. Queloz ${ }^{1}$, D. Ségransan ${ }^{1}$, B. Smalley ${ }^{2}$, \\ A. M. S. Smith ${ }^{2,6}$, S. Udry ${ }^{1}$, R. G. West ${ }^{7}$, and P. J. Wheatley ${ }^{5}$ \\ ${ }^{1}$ Observatoire Astronomique de l'Université de Genève, Chemin des Maillettes 51, 1290 Sauverny, Switzerland \\ e-mail: Amaury.Triaud@unige.ch \\ 2 Astrophysics Group, Keele University, Staffordshire, ST55BG, UK \\ 3 SUPA, School of Physics \& Astronomy, University of St. Andrews, North Haugh, KY16 9SS, St. Andrews, Fife, Scotland, UK \\ ${ }^{4}$ Institut d'Astrophysique et de Géophysique, Université de Liège, Allée du 6 Août, 17, Bat. B5C, Liège 1, Belgium \\ 5 Department of Physics, University of Warwick, Coventry CV4 7AL, UK \\ ${ }^{6}$ N. Copernicus Astronomical Centre, Polish Academy of Sciences, Bartycka 18, 00-716 Warsaw, Poland \\ 7 Department of Physics and Astronomy, University of Leicester, Leicester, LE17RH, UK
}

Received 13 December 2012 / Accepted 9 January 2013

\section{ABSTRACT}

\begin{abstract}
We report the discovery of a planet transiting the star WASP-80 (1SWASP J201240.26-020838.2; 2MASS J20124017-0208391; TYC 5165-481-1; BPM 80815; $V=11.9, K=8.4$ ). Our analysis shows this is a $0.55 \pm 0.04 M_{\text {jup }}, 0.95 \pm 0.03 R_{\text {jup }}$ gas giant on a circular 3.07 day orbit around a star with a spectral type between K7V and M0V. This system produces one of the largest transit depths so far reported, making it a worthwhile target for transmission spectroscopy. We find a large discrepancy between the $v \sin i_{\star}$ inferred from stellar line broadening and the observed amplitude of the Rossiter-McLaughlin effect. This can be understood either by an orbital plane nearly perpendicular to the stellar spin or by an additional, unaccounted for source of broadening.
\end{abstract}

Key words. planetary systems - binaries: eclipsing - stars: individual: WASP-80 - techniques: radial velocities techniques: photometric - techniques: spectroscopic

\section{Introduction}

Numerous planets have been found since Mayor \& Queloz (1995) and, like 51 Pegasi b, most orbit stars whose spectral type, mass, or size are similar to the Sun's. This occurs even though a few surveys have concentrated their efforts on other spectral types notably towards M dwarfs, such as the HARPS M-dwarf survey (Bonfils et al. 2013) or the M-Earth project (Nutzman \& Charbonneau 2008). In their rarity, those planets nevertheless help us better understand the processes leading to planet formation.

The Wide Angle Search for Planets (WASP) survey aims to find transiting planets (Pollacco et al. 2006), and has now surveyed most of the night sky in both hemispheres. With some 70 planets now publicly announced, this is the most efficient ground-based planet discovery project. Thanks to its observation of now more than 30 million stars of magnitude between 8.5 and 13.5 , it can pick up those rare planets that have avoided detection by the radial-velocity surveys or even by the space-missions Kepler and CoRoT, which have surveyed only 150000 stars each. Amongst those rare planets found by

* Using WASP-South photometric observations, from Sutherland (South Africa), confirmed with the $60 \mathrm{~cm}$ TRAPPIST robotic telescope, EulerCam, and the CORALIE spectrograph on the Swiss $1.2 \mathrm{~m}$ Euler Telescope, and HARPS on the ESO $3.6 \mathrm{~m}$ (Prog ID 089.C-0151), all three located at La Silla Observatory, Chile.

$\star \star$ Radial velocity and photometric data are available in electronic form at the CDS via anonymous ftp to

cdsarc.u-strasbg.fr (130.79.128.5) or via

http://cdsarc.u-strasbg.fr/viz-bin/qcat?]/A+A/551/A80
WASP is the first gas giant around a $\delta$ Scuti (Collier Cameron et al. 2010) and the population of very short period gas giants, such as WASP-12, 18, 19, and 43 (Hebb et al. 2009, 2010; Hellier et al. 2009, 2011).

Despite their numbers and the facility of discovering them (radial velocities or transits), the occurrence rate of hot Jupiters orbiting solar-type stars is low. It has been estimated to be as high as $1.5 \pm 0.6 \%$ by Cumming et al. (2008) from radial velocity surveys, and as low as $0.5 \pm 0.1 \%$ by Howard et al. (2012) from the Kepler results. Johnson et al. (2010) have made a case that, because no hot Jupiter was known to orbit an M dwarf, their occurrence must therefore be lower. Not long afterwards, Johnson et al. (2012) announced the discovery of a transiting gas giant around a star observed by Kepler, KOI-254, describing it as a "lone example [...] for some time to come". Approximately $300 \mathrm{M}$ dwarf systems have been searched for planets between the main radial velocity teams (Johnson et al. 2010). The M-Earth project is targeting about 3000 (with a geometrical detection of only $5-10 \%$ ). If the rate of hot Jupiters is but a half to a third that of solar type stars, there is a significant chance that such planets have avoided detection, a point made by Bonfils et al. (2013). Knowing this rate is important since gas giant formation is perceived as less efficient because protoplanetary disc masses scale with their primary's mass as dynamical timescales do (Laughlin et al. 2004; Ida \& Lin 2005; Alibert et al. 2011; Mordasini et al. 2012).

Within this context, we announce the discovery of a gas giant transiting a late K-early M dwarf. We first describe our data collection, then its analysis, and finally the results we obtain. 

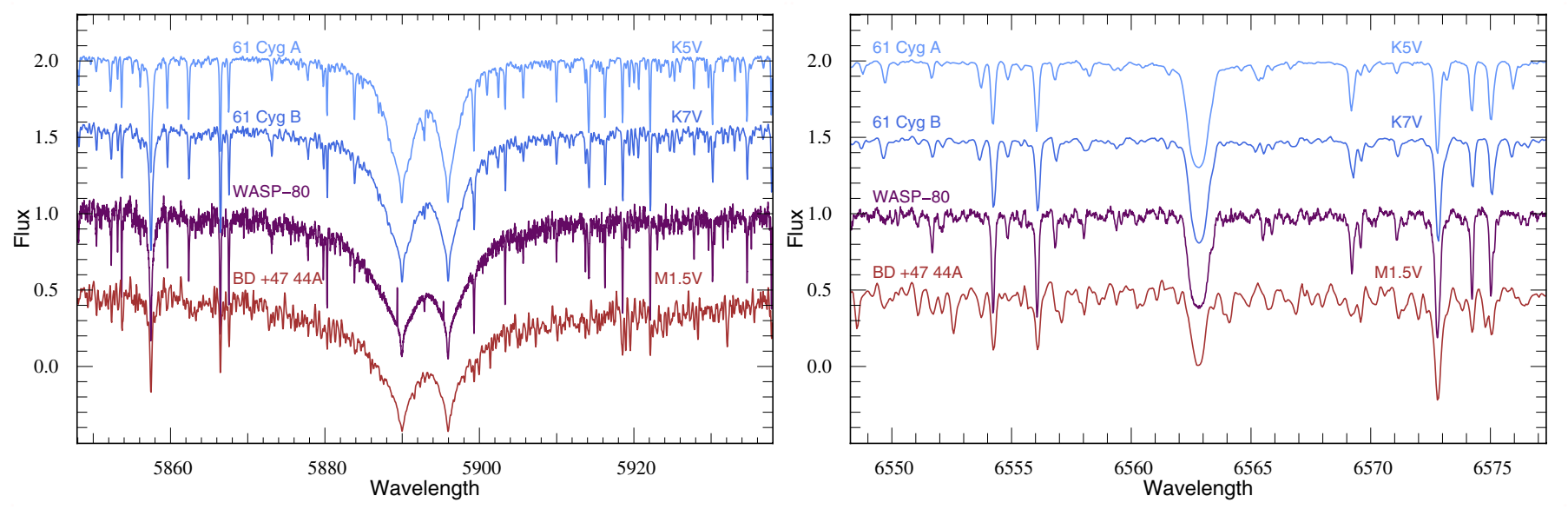

Fig. 1. Co-added HARPS spectra on WASP-80 compared with ELODIE spectra of $61 \mathrm{Cyg}$ A \& B and of BD $+4744 \mathrm{~A}$ over the Na I Doublet and $\mathrm{H}_{\alpha}$.

\section{Observations}

WASP-80 (1SWASP J201240.26-020838.2;2MASS J201240170208391; TYC 5165-481-1; BPM 80815) was observed 5782 times during one season at the WASP-South facility in Sutherland (South Africa), by a single camera, between 2010 May 15 and 2010 September 26 (Fig. 2b). The Hunter algorithm (Collier Cameron et al. 2007) found a period at 3.07 days and uncovered a transit-like signal from five partial events, with a large depth that nevertheless corresponds to a planet-sized object once the colours of the star indicated it was a potential M0 dwarf.

The star was catalogued for spectroscopic follow-up on 2011 May 09, and the first radial velocity measurements were obtained with the CORALIE spectrograph in July 2011. Thirty-seven spectra have been collected between 2011 July 21 and 2012 September 12, including ten measurements obtained during the Rossiter-McLaughlin effect on 2012 June 19. We also used HARPS and acquired sixteen spectra during the transit of 2012 September 10. Atmospheric conditions were poor with seeing $>2^{\prime \prime}$ at the beginning of the sequence. Eight measurements were obtained in the nights leading to and following the transit, with one point badly affected by weather and excluded from the analysis. Radial velocities were extracted using a K5 correlation mask, and those data also show a 3.07 day variation, in phase with the photometry. No such variation can be observed in the span of the bisector slope, or into the width of the line (Fig. 2a). This indicates a movement of the spectrum with time, as expected for an orbiting planet.

To complete the confirmation of the system and obtain precise physical parameters, three higher precision lightcurves were observed, two by TRAPPIST (Jehin et al. 2011) on 2012 May 07 and 2012 September 10 in the $z$-band, and one by EulerCam using an r'-Gunn filter on 2012 July 26 (Fig. 2c). Our data were reduced and prepared for analysis in the same manner as in previous WASP discoveries. Useful references can be found in Wilson et al. (2008), Anderson et al. (2011), Gillon et al. (2009, 2012), and Lendl et al. (2012).

\section{Spectral analysis}

The HARPS spectra were co-added, leading to a single spectrum of signal-to-noise of 75:1. Its analysis was conducted following the methods described in Doyle et al. (2013). The results are displayed in Table 1. The $\mathrm{H}_{\alpha}$ line being weak, it indicates a low effective temperature around $4000 \mathrm{~K}$ and a $\mathrm{K} 7$ spectral type. The TiO bands are also weak, typical of a late K-early $\mathrm{M}$ dwarf. The $\mathrm{Na}$ I $\mathrm{D}$ are very strong, implying a surface gravity $(\log g)$ around 4.6 , closer to an M spectral type than a K7 (Fig. 1). The equivalent widths of several clean and unblended Fe I lines were measured, in order to determine the stellar metallicity, evaluate microturbulence, and confirm the $T_{\text {eff }}$ estimated from $\mathrm{H}_{\alpha}$. They were also fitted to estimate the broadening caused by the projected stellar rotation velocity $\left(v \sin i_{\star}\right)$. Macroturbulence was assumed to be zero since its effect is expected to be lower than thermal broadening (Gray 2008). We found $v \sin i_{\star}=3.55 \pm 0.33 \mathrm{~km} \mathrm{~s}^{-1}$. Because this seemed unusually large for this spectral type, we looked at all the stars with a similar $B-V$ present in the HARPS archive: WASP- 80 has the widest lines in the sample. There is no significant detection of lithium in the spectrum, and we can place an equivalent-width upper limit of $30 \mathrm{~m} \AA$, meaning $\log A(\mathrm{Li})<0.0 \pm 0.2$. For early M-type stars, lithium can be this depleted in less than $100 \mathrm{My}$ (Sestito \& Randich 2005).

In their survey of high proper motion stars, Stephenson (1986) listed the star as spectral type K5. This is, however, inconsistent with the results of the spectral analysis. An independent check of the stellar temperature can be obtained from the infrared flux method (IRFM, Blackwell \& Shallis 1977), which has been used to determine $T_{\text {eff }}$ and stellar angular diameter $(\theta)$ by estimating the total observed bolometric flux from broadband photometry from NOMAD, TASS, CMC14, and 2MASS. This gives $T_{\text {eff }}=4020 \pm 130 \mathrm{~K}$ and $\theta=0.113 \pm 0.008$ mas, consistent with the $T_{\text {eff }}$ from the spectral analysis. There is no sign of any interstellar Na D lines in the spectra, so reddening is expected to be negligible.

\section{Results}

We applied the same fitting Markov chain Monte Carlo (MCMC) algorithm as described in Triaud et al. (2011). The stellar mass was constrained by a Gaussian prior with mean and standard deviation corresponding to the value obtained using the empirical mass-radius relation from Torres et al. (2010). The fit of the model over the data informs us of the mean stellar density (Seager \& Mallén-Ornelas 2003) found to be $3.12 \pm 0.02 \rho_{\odot}$. When combined with a mass of $0.58 \pm 0.05 M_{\odot}$ it gives a radius of $0.57 \pm 0.02 R_{\odot}$ (entirely compatible with the Torres relation) and a $\log g_{\star}=4.69 \pm 0.02$. Those values are also compatible with theoretical mass-radius relationships presented in Baraffe et al. (1998, see Fig. 3). 

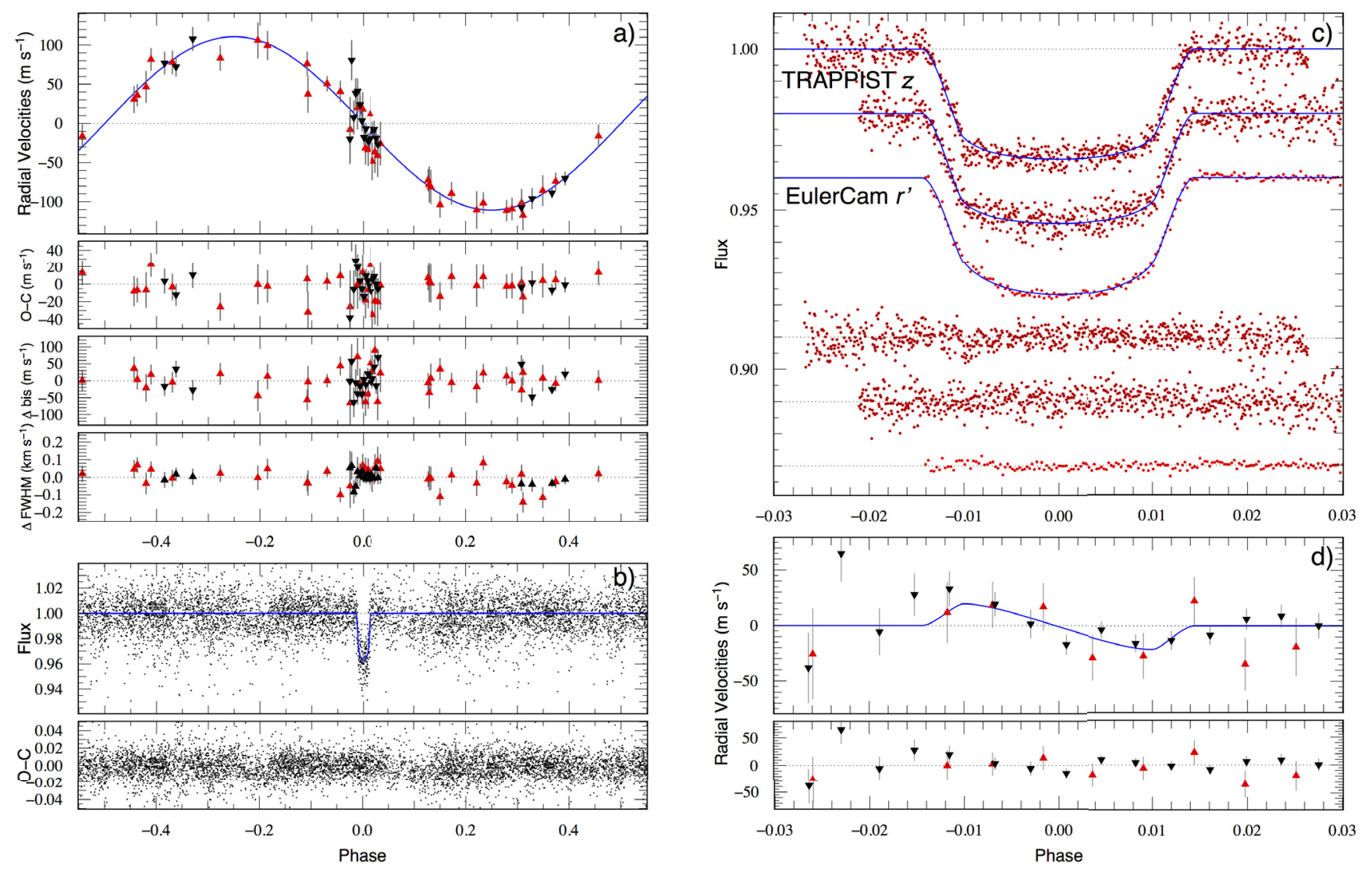

Fig. 2. a) Top to bottom: CORALIE (upright red triangles) and HARPS (inverted black triangles) radial velocities on WASP-80 plotted with a circular Keplerian model and residuals; below: change in the span of the bisector slope and change in the FWHM of the CCF. b) Phase-folded WASP V+R photometry with model and its residuals. c) Top to bottom: the two TRAPPIST $z$ band and the EulerCam r'-Gunn transit lightcurves with models over plotted. The residuals are displayed in the same order below. d) Zoom on the Rossiter-McLaughlin effect showing CORALIE and HARPS radial velocities with the most likely model and the residuals from the fit.

The fit to the radial-velocities gives a reduced $\chi_{\mathrm{r}}^{2}=0.75 \pm$ 0.17 , suggesting we may have overestimated our error bars on the measurements. The largest contribution to the $\chi^{2}$ comes from the second point in the HARPS Rossiter-McLaughlin series, likely affected by high seeing. No eccentricity can be detected. We place a 95\% confident upper value at $e<0.07$. Similarly, if there is any other perturber in the system, it adds an acceleration lower than $24 \mathrm{~m} \mathrm{~s}^{-1} \mathrm{yr}^{-1}$. The results of the fit are located in Table 1 . The mass function $\mathrm{f}(\mathrm{m})$ and the $\log g_{\mathrm{p}}$ are directly obtained from fitting the data. They indicate we have discovered a new transiting planet. Using the stellar mass we obtain a mass and radius for our object and find $0.55 \pm 0.04 M_{\text {jup }}$ and $0.95 \pm 0.03 R_{\text {jup }}$.

The Rossiter-McLaughlin effect is marginally detected, even though a semi amplitude between 60 and $70 \mathrm{~m} \mathrm{~s}^{-1}$ had been expected. This can be explained either by a highly inclined planet (the impact parameter $b<0.1$, even when adjusting only the photometry (see Triaud et al. 2011 for details) or by an additional unaccounted-for broadening of the spectral lines. This could occur in the presence of magnetic fields producing a partially resolved Zeeman line splitting. The presence of strong magnetic fields has been reported for a number of $\mathrm{M}$ dwarfs by Morin et al. (2010) and Donati et al. (2008). We examined our spectra and found no signs of Zeeman broadening.

Two different fits were attempted. For the first one, we assumed $\beta=0^{\circ} \pm 20$ and found $V \sin i_{\star}=0.91 \pm 0.25 \mathrm{~km} \mathrm{~s}^{-1}$, in strong disagreement with the value inferred in Sect. 3. For the second attempt, we chose to impose a prior on $V \sin i_{\star}=v \sin i_{\star}$, and as expected we find two well separated and symmetrical solutions for the spin-orbit angle: $\beta= \pm 75^{\circ} \pm 4$. We caution here that this angle is entirely dependent on the value of $v \sin i_{\star}$.

The rotation rate $(P=8.5 \pm 0.8 \mathrm{~d})$ implied by the $v \sin i_{\star}$ gives a gyrochronological age of $\sim 100_{-20}^{+30}$ My using the Barnes (2007) relation. The presence of $\mathrm{Ca} \mathrm{H}+\mathrm{K}$ emission indicates that WASP-80 may be a young active star. Despite this, we do not detect any rotational variability $>1$ mmag in the WASP lightcurve or in a multi-epoch TRAPPIST campaign. None of the transits show signs of stellar spot crossings.

Furthermore, using the values of proper motion reported in NOMAD (Zacharias et al. 2004) $\left(-100 \pm 3,-60 \pm 8{\left.\text { mas } \mathrm{yr}^{-1}\right)}^{-1}\right.$ and the systemic velocity we observed $\left(10.2 \mathrm{~km} \mathrm{~s}^{-1}\right)$, we computed its galactic dynamical velocities $(U, V, W)=(30.0,-11.7$, $12.8) \mathrm{km} \mathrm{s}^{-1}$. Those values are well away from any of the known young moving groups reported in Zuckerman \& Song (2004). This means the gyrochronological age is not reliable.

\section{Conclusions}

Our observations and their analysis allow us to conclude there is an unseen transiting companion orbiting WASP-80 whose mass and radius are planetary. Even though only a few cool stars have been observed by WASP, our planet confirmation rate is similar to Sun-like stars. We only followed-up 26 stars for which the Hunter algorithm returned a signal and whose colours indicate they are of K5 or later type (only three were classified as M0 including WASP-80). Our data show we have 17 blends (for eight of those the original target was identified as a red 
Table 1. Parameters of the WASP-80 system.

\begin{tabular}{|c|c|c|c|}
\hline \multicolumn{4}{|c|}{$\begin{array}{c}\text { 1SWASP J201240.26-020838.2 } \\
\text { 2MASS J20124017-0208391 } \\
\text { TYC 5165-481-1 } \\
\text { BPM 80815 }\end{array}$} \\
\hline Filter & Magnitude & Filter & Magnitude \\
\hline $2 M A S S^{1}$ & \multicolumn{3}{|c|}{$N_{O M A D^{2}}$} \\
\hline$J$ & $9.218 \pm 0.023$ & $B$ & 12.810 \\
\hline$H$ & $8.513 \pm 0.026$ & $V$ & 11.870 \\
\hline K & $8.351 \pm 0.022$ & $R$ & 11.110 \\
\hline$T A S S 4^{3}$ & \multicolumn{3}{|c|}{$\mathrm{CMC14^{4 }}$} \\
\hline$V$ & $11.881 \pm 0.228$ & $r^{\prime}$ & $11.358 \pm 0.011$ \\
\hline I & \multicolumn{3}{|l|}{$10.279 \pm 0.105$} \\
\hline Parameter & Value \& $1 \sigma$ error & Parameter & Value \& $1 \sigma$ error \\
\hline \multicolumn{4}{|c|}{ from spectral line analysis } \\
\hline Spectral type & $\mathrm{K} 7 \mathrm{~V}$ & Distance & $60 \pm 20 \mathrm{pc}$ \\
\hline$T_{\text {eff }}$ & $4145 \pm 100 \mathrm{~K}$ & {$[\mathrm{Fe} / \mathrm{H}]$} & $-0.14 \pm 0.16$ \\
\hline $\log g$ & $4.6 \pm 0.2(\mathrm{cgs})$ & $\log \mathrm{A}(\mathrm{Li})$ & $<0.0 \pm 0.2$ \\
\hline$v \sin i_{\star}$ & $3.55 \pm 0.33 \mathrm{~km} \mathrm{~s}^{-1}$ & $\xi_{\mathrm{t}}$ & $0.3 \pm 0.3 \mathrm{~km} \mathrm{~s}^{-1}$ \\
\hline Mass & $0.58 \pm 0.05 M_{\odot}$ & Radius & $0.63 \pm 0.15 R_{\odot}$ \\
\hline \multicolumn{4}{|c|}{ jump parameters for the MCMC } \\
\hline Period & $3.0678504 \underset{(-27)}{(+23)} \mathrm{d}$ & $T_{0}(\mathrm{BJD})$ & $2456125.417512_{(-52)}^{(+67)}$ \\
\hline Depth & $0.02933_{(-09)}^{(+10)}$ & Width & $0.08800{ }_{(-16)}^{(+19)} d$ \\
\hline$\sqrt{V \sin i_{\star}} \cos \beta$ & $0.48_{(-0.13)}^{(+0.12)}$ & $b$ & $0.019_{(-17)}^{(+26)} R_{\odot}$ \\
\hline$\sqrt{V \sin i_{\star}} \sin \beta$ & $\pm 1.78_{(-0.09)}^{(+0.12)}$ & $K$ & $110.9_{(-3.3)}^{(+3.0)} \mathrm{m} \mathrm{s}^{-1}$ \\
\hline \multicolumn{4}{|c|}{ derived parameters from the $M C M C$} \\
\hline$R_{\mathrm{p}} / R_{\star}$ & $0.17126_{(-26)}^{(+31)}$ & $f(m)$ & $0.425 \times 10^{-9} \underset{(-31)}{(+43)} M_{\odot}$ \\
\hline$R_{\star} / a$ & $0.07699_{(-17)}^{(+17)}$ & $R_{\mathrm{p}} / a$ & $0.013183_{(-35)}^{(+39)}$ \\
\hline $\log g_{\star}$ & $4.689_{(-13)}^{(+12)}(\mathrm{cgs})$ & $\log g_{\mathrm{p}}$ & $3.178_{(-12)}^{(+13)}(\mathrm{cgs})$ \\
\hline$\rho_{\star}$ & $3.117_{(-20)}^{(+21)} \rho_{\odot}$ & $\rho_{\mathrm{p}}$ & $0.554_{(-39)}^{(+30)} \rho_{\text {jup }}$ \\
\hline$M_{\star}$ (prior) & $0.57_{(-0.05)}^{(+0.05)} M_{\odot}$ & $M_{\mathrm{p}}$ & $0.554_{(-39)}^{(+30)} M_{\text {jup }}$ \\
\hline$R_{\star}$ & $0.571_{(-16)}^{(+16)} R_{\odot}$ & $R_{\mathrm{p}}$ & $0.952_{(-27)}^{(+26)} R_{\text {jup }}$ \\
\hline$V \sin i_{\star}$ & $3.46_{(-0.35)}^{(+0.34)} \mathrm{km} \mathrm{s}^{-1}$ & $\beta$ & $\pm 755_{(-4.3)}^{(+4.0)} \mathrm{deg}$ \\
\hline$a$ & $0.0346_{(-11)}^{(+08)} \mathrm{AU}$ & $i_{\mathrm{p}}$ & $89.92_{(-0.12)}^{(+0.07)} \mathrm{deg}$ \\
\hline$e$ & $<0.07$ & $|\dot{\gamma}|$ & $<24 \mathrm{~m} \mathrm{~s}^{-1} \mathrm{yr}^{-1}$ \\
\hline
\end{tabular}

Notes. Mass and radius estimated using the Torres et al. (2010) calibration. Spectral type estimated from $T_{\text {eff }}$ using Table B.1 in Gray (2008). Distance estimated using the IRFM angular diameter and the stellar radius. Units based on the equatorial solar and jovian radii and masses taken from Allen's Astrophysical Quantities (Cox 2000)

References. 1 - Skrutskie et al. (2006); 2 - Zacharias et al. (2004); 3 - Droege et al. (2006); 4 - ViZier I/304/out.

giant), three spectrally resolved eclipsing binaries and one unresolved, two potential triples (blends but gravitationally bound), one false alarm, and two planets (WASP-43b (Hellier et al. 2011; Gillon et al. 2012), and WASP-80b). A total of two planets out of 26 candidates is remarkably close to our mean discovery rate of $8.8 \pm 1.2 \%$ planet per candidate (Triaud 2011). In addition to the observed rarity of hot Jupiters around cold stars, it is also interesting to note that this planet is orbiting a relatively metal poor star, whereas Santos et al. (2003), Fischer \& Valenti (2005), and Mayor et al. (2011) have shown they have an even lower occurrence rate of gas giants for any orbital period.

WASP-80b's equilibrium temperature will be around $800 \mathrm{~K}$ (for an albedo of 0.1). The planet-to-star contrast is favourable

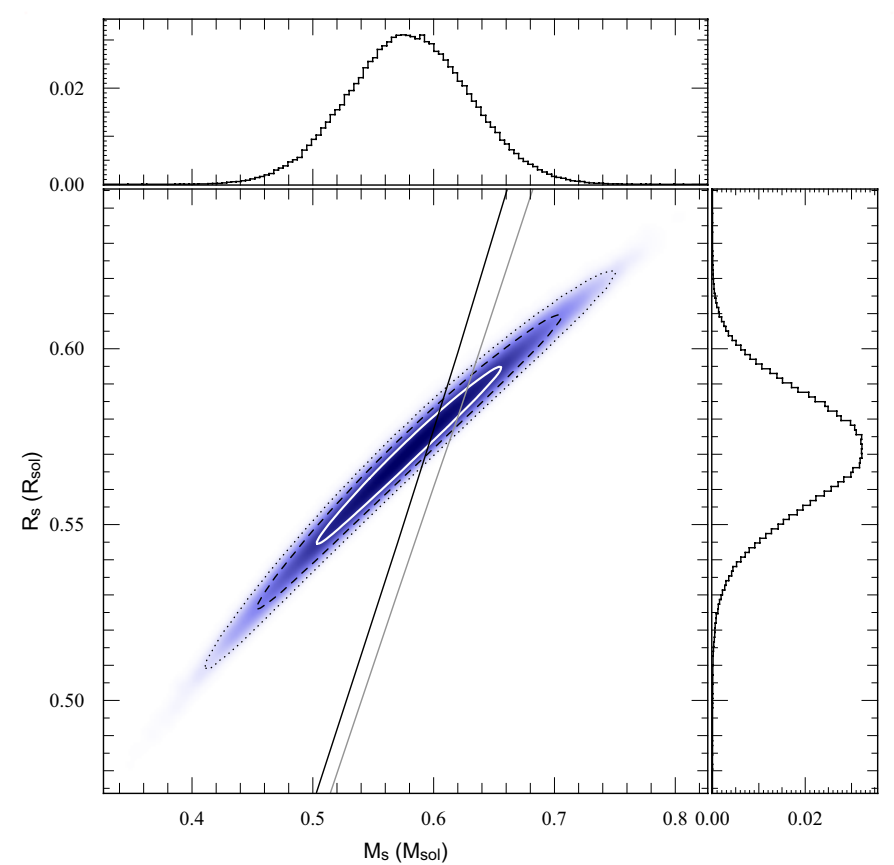

Fig. 3. Posterior probability density, output of the MCMC, showing the mass of the primary (drawn from a prior), and its radius (inferred from the transit signal). 1,2, and $3 \sigma$ confidence regions are drawn. The overplotted grey line is the theoretical 0.5 Gyr mass-radius relationship from Baraffe et al. (1998), in black is the 8 Gyr relation (for solar metallicity). Side histograms show the marginalised parameters.

for future observation of the emission spectrum of the planet, because it is hosted by a star $\sim 1500 \mathrm{~K}$ colder than the usual targets. Furthermore, the near 3\% depth of the transit makes this gas giant one of the most suitable targets for transmission spectroscopy.

WASP-80b is a warm Jupiter when we consider its temperature, and yet it belongs to the hot Jupiter population. Because of the high density of the host, and the low density of the planet, it is located about 3 Roche radii away from the star, just as would be expected if it had circularised from an earlier, more eccentric orbit (Matsumura et al. 2010). Although the Rossiter-McLaughlin effect is observed symmetrical, the planet's orbital spin could be severely inclined if the host star rotates as quickly as the spectral line broadening indicates. If no additional spectral line broadening mechanism is discovered, then WASP-80b will become a rare example of a severely inclined planet whose host star's $T_{\text {eff }}$ is cooler than $6250 \mathrm{~K}$ (Brown et al. 2012; Albrecht et al. 2012; Winn et al. 2010).

Nota Bene We used the UTC time standard and barycentric Julian dates in our analysis.

Acknowledgements. We acknowledge an extensive use of Harvard's ADS paper archive and Strasbourg's CDS data repository. We would like to thank Richard I. Anderson for his kindness in observing the CORALIE Rossiter-McLaughlin effect, with quite late notice and under a certain weather stress, as well as to thank Xavier Bonfils and Corinne Charbonnel for inspiring discussions. This work is supported by the Swiss National Science Foundation. TRAPPIST is a project funded by the Belgian Fund for Scientific Research (Fond National de la Recherche Scientifique, F.R. SFNRS) under grant FRFC 2.5.594.09.F, with the participation of the Swiss National Science Foundation (SNF). M. Gillon and E. Jehin are FNRS Research Associates. WASP-South is hosted by the South African Astronomical Observatory, and we are grateful for their ongoing support and assistance. This publication makes use of data products from the Two Micron All Sky Survey, which is a joint project of the University of Massachusetts and the Infrared Processing and Analysis Center/California Institute of Technology, funded by the National Aeronautics and Space Administration and the National Science Foundation. 


\section{A. H. M. J. Triaud et al.: WASP-80b}

\section{References}

Albrecht, S., Winn, J. N., Johnson, J. A., et al. 2012, ApJ, 757, 18

Alibert, Y., Mordasini, C., \& Benz, W. 2011, A\&A, 526, A63

Anderson, D. R., Collier Cameron, A., Hellier, C., et al. 2011, A\&A, 531, A60

Baraffe, I., Chabrier, G., Allard, F., \& Hauschildt, P. H. 1998, A\&A, 337, 403

Barnes, S. A. 2007, ApJ, 669, 1167

Blackwell, D. E., \& Shallis, M. J. 1977, MNRAS, 180, 177

Bonfils, X., Delfosse, X., Udry, S., et al. 2013, A\&A, 549, A109

Brown, D. J. A., Cameron, A. C., Anderson, D. R., et al. 2012, MNRAS, 423, 1503

Collier Cameron, A., Wilson, D. M., West, R. G., et al. 2007, MNRAS, 380, 1230

Collier Cameron, A., Guenther, E., Smalley, B., et al. 2010, MNRAS, 407, 507

Cox, A. N. 2000, Allen's astrophysical quantities (New York: AIP Press; Springer)

Cumming, A., Butler, R. P., Marcy, G. W., et al. 2008, PASP, 120, 531

Donati, J.-F., Morin, J., Petit, P., et al. 2008, MNRAS, 390, 545

Doyle, A. P., Smalley, B., Maxted, P. F. L., et al. 2013, MNRAS, 428, 3164

Droege, T. F., Richmond, M. W., Sallman, M. P., \& Creager, R. P. 2006, PASP, 118,1666

Fischer, D. A., \& Valenti, J. 2005, ApJ, 622, 1102

Gillon, M., Anderson, D. R., Triaud, A. H. M. J., et al. 2009, A\&A, 501, 785

Gillon, M., Triaud, A. H. M. J., Fortney, J. J., et al. 2012, A\&A, 542, A4

Gray, D. F. 2008, The Observation and Analysis of Stellar Photospheres (Cambridge, UK: Cambridge University Press)

Hebb, L., Collier-Cameron, A., Loeillet, B., et al. 2009, ApJ, 693, 1920

Hebb, L., Collier-Cameron, A., Triaud, A. H. M. J., et al. 2010, ApJ, 708, 224

Hellier, C., Anderson, D. R., Collier Cameron, A., et al. 2009, Nature, 460, 1098

Hellier, C., Anderson, D. R., Collier Cameron, A., et al. 2011, A\&A, 535, L7

Howard, A. W., Marcy, G. W., Bryson, S. T., et al. 2012, ApJS, 201, 15
Ida, S., \& Lin, D. N. C. 2005, ApJ, 626, 1045

Jehin, E., Gillon, M., Queloz, D., et al. 2011, The Messenger, 145, 2

Johnson, J. A., Howard, A. W., Marcy, G. W., et al. 2010, PASP, 122, 149

Johnson, J. A., Gazak, J. Z., Apps, K., et al. 2012, AJ, 143, 111

Laughlin, G., Bodenheimer, P., \& Adams, F. C. 2004, ApJ, 612, L73

Lendl, M., Anderson, D. R., Collier-Cameron, A., et al. 2012, A\&A, 544, A72

Matsumura, S., Peale, S. J., \& Rasio, F. A. 2010, ApJ, 725, 1995

Mayor, M., \& Queloz, D. 1995, Nature, 378, 355

Mayor, M., Marmier, M., Lovis, C., et al. 2011, A\&A, submitted [arXiv: 1109.2497]

Mordasini, C., Alibert, Y., Benz, W., Klahr, H., \& Henning, T. 2012, A\&A, 541, A97

Morin, J., Donati, J.-F., Petit, P., et al. 2010, MNRAS, 407, 2269

Nutzman, P., \& Charbonneau, D. 2008, PASP, 120, 317

Pollacco, D. L., Skillen, I., Collier Cameron, A., et al. 2006, PASP, 118, 1407

Santos, N. C., Israelian, G., Mayor, M., Rebolo, R., \& Udry, S. 2003, A\&A, 398 363

Seager, S., \& Mallén-Ornelas, G. 2003, ApJ, 585, 1038

Sestito, P., \& Randich, S. 2005, A\&A, 442, 615

Skrutskie, M. F., Cutri, R. M., Stiening, R., et al. 2006, AJ, 131, 1163

Stephenson, C. B. 1986, AJ, 92, 139

Torres, G., Andersen, J., \& Giménez, A. 2010, A\&ARv, 18, 67

Triaud, A. H. M. J. 2011, Ph.D. Thesis, Observatoire Astronomique de l'Université de Genève http://archive-ouverte.unige.ch/unige: 18065

Triaud, A. H. M. J., Queloz, D., Hellier, C., et al. 2011, A\&A, 531, A24

Wilson, D. M., Gillon, M., Hellier, C., et al. 2008, ApJ, 675, L113

Winn, J. N., Fabrycky, D., Albrecht, S., \& Johnson, J. A. 2010, ApJ, 718, L145

Zacharias, N., Monet, D. G., Levine, S. E., et al. 2004, in BAAS, 36, Am. Astron. Soc. Meeting Abstracts, 1418

Zuckerman, B., \& Song, I. 2004, ARA\&A, 42, 685 